\section{Keeping it in the family}

It is well established that maternal stress and nutrition during pregnancy can influence the developing fetus, but recently there has been a surge of interest in the possible transfer of paternal experiences to the offspring. Bale and colleagues now show that paternal exposure to stress influences stress responsiveness in the offspring, possibly through changes in the microRNA (miRNA) content of sperm.

The authors exposed male mice to 6 weeks of chronic variable stress, either during puberty or during adulthood. Two weeks post-stress, the males were allowed to mate with females, and the authors assessed the resulting offspring for sensitivity to stress in adulthood. Both male and female offspring of males that were chronically stressed in puberty or adulthood showed a suppression of hypothalamus-pituitary-adrenal (HPA) axis activity in response to acute restraint stress. These effects were specific for the hormonal response to stress, as there were no effects in behavioural tests of stress responsiveness.

The authors next set out to identify the mediators of the HPA axis activity in the offspring of stressed males. They found that there was no effect of paternal stress on serotonin regulation of the HPA axis or on the expression of several known peripheral regulators of HPA axis activity in these offspring.
By contrast, gene expression in two brain areas known to regulate HPA axis activity, the hypothalamic paraventricular nucleus (PVN) and the bed nucleus of the stria terminalis (BNST), showed robust alterations induced by paternal stress. Gene set enrichment analysis of microarray data from these areas revealed upregulation of several gene sets in male and female offspring of stressed males, indicating global, probably epigenetic, reprogramming. In the PVN, this involved $\mathrm{C} 3$ gene sets (members of a particular C3 set are regulated by the same transcription factor or miRNA), some of which

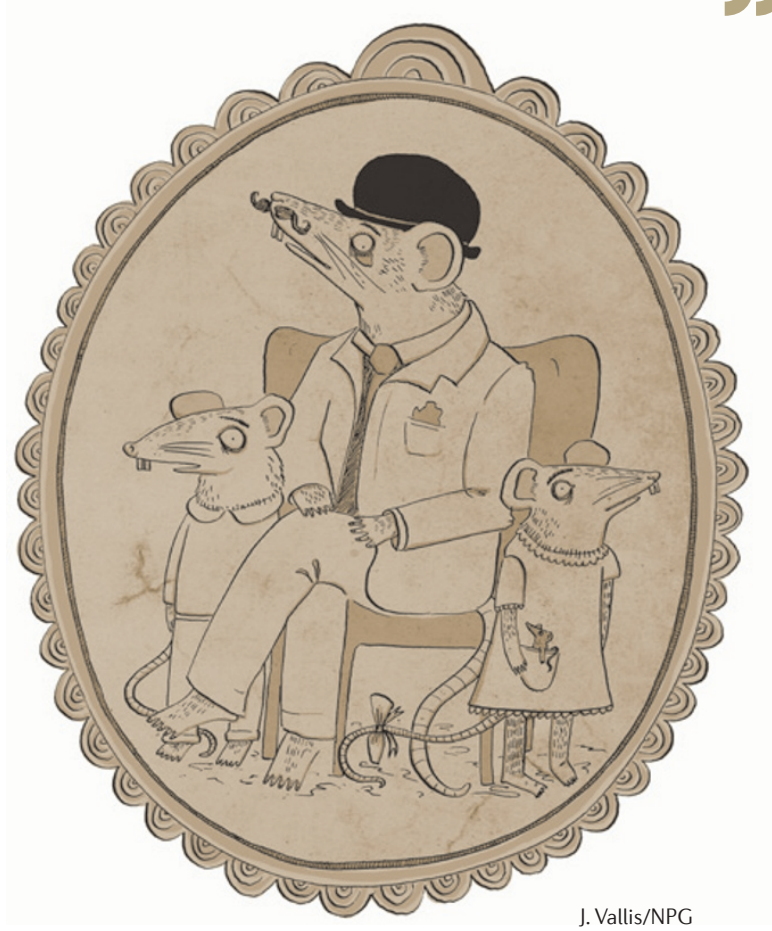

included glucocorticoid-responsive genes. The BNST showed enrichment of many $\mathrm{C} 3$ gene sets as well as $\mathrm{C} 5$ gene sets (members of which have a common ontology but are not necessarily co-regulated), and these included genes involved in chromatin modification, neuronal survival, gene expression and a large number of miRNA-encoding genes.

As previous studies suggested that epigenetic changes in sperm may underlie the transmission of paternal experiences to the offspring, the authors analysed the miRNA content of sperm from stressed males. This revealed that stress exposure in puberty or adulthood produced a distinct global pattern of miRNA expression, with significant increases in the expression of nine specific miRNAs, the predicted targets of which include an RNA encoding a DNA methyltransferase and RNAs encoding proteins involved in miRNA processing.

These findings show that stress exposure in males can induce longterm epigenetic reprogramming of germ cells, and that this may provide a mechanism by which paternal experiences can influence neurodevelopment and stress responsiveness in the offspring.

Leonie Welberg

ORIGINAL RESEARCH PAPER Rodgers, A. B. et al.

Paternal stress exposure alters sperm microRNA content and reprograms offspring HPA stress axis regulation. J. Neurosci. 33, 9003-9012 (2013) 